\title{
Can CRP affect the blood-brain barrier during acute ischemic stroke?
}

\begin{abstract}
Introduction. Ischemic strokes (IS) are one of the main causes of death and disabilities around the globe. Therefore, there is a huge need for researching the pathogenesis of IS. The C-reactive protein (CRP) plays a role during inflammatory processes. Results of some studies conducted on animal models indicate that CRP affects the blood-brain barrier (BBB) stability during IS. The presence of S100BB protein can be considered as an indication of BBB injury.

Aim. The purpose of this study was to discover the relationship between CRP and S100BB protein.

Material and methods. The study looked at fifty four IS patients, with the disease confirmed by computer tomography (CT). The clinical status was evaluated on the $1^{\text {st }}, 3^{\text {rd }}, 5^{\text {th }}, 10^{\text {th }}$ day and 3 months following the onset of IS. Neurological status was estimated using the National Institute of Health Stroke Scale (NIHSS). Patients' disability level was determined, using Modified Rankin Scale (mRS) and Barthel Index (BI). The volume of ischemic focus was calculated on the $10^{\text {th }}$ day after the stroke, using CT. The levels of CRP and S100BB were evaluated on $1^{\text {st }}, 3^{\text {rd }}, 5^{\text {th }}$ and $10^{\text {th }}$ day after the stroke onset with usage of ELISA method.

Results. The mean level of CRP and its concentration on the $1^{\text {st }}, 3^{\text {rd }}, 5^{\text {th }}$ and $10^{\text {th }}$ day directly correlates with a deteriorated clinical status, as measured with the use of NIHSS, BI and mRS on day 10 and 3 months after the onset of IS. We found a correlation with the mean CRP level and bigger volume of ischemic injury. The mean CRP level correlated with the mean level of S100BB protein. In the group of patients with low CRP $(0.51-24.68 \mathrm{mg} / \mathrm{mL})$ the level of S100BB and the volume of ischemic focus were lower than in the group with a high level of CRP $(24.69-209 \mathrm{mg} / \mathrm{mL})$.

Conclusions. CRP can be considered as a predictor of a worse clinical outcome after stroke. The relationship between CRP and S100BB protein can suggest the active role of CRP during IS.
\end{abstract}

Keywords: CRP, ischemic stroke, S100BB protein, blood-brain barrier.

DOI: $10.1515 /$ pjph-2015-0033

\section{INTRODUCTION}

Ischemic stroke (IS) is one of the leading causes of death and patients' disability worldwide. Numerous past investigations looking at IS pathogenesis give hope of a discovery of better and more effective therapeutic methods. C-reactive protein (CRP) belongs to the family of acute-phase proteins. It is produced in the liver as a response to factors released by adipocytes and macrophages, mainly cytokine interleukin (IL)-6, during inflammatory processes. The function of CRP is to enhance phagocytosis by macrophages by binding to phosphocholine on the surface of dying cells in order to activate the complement system $[1,2]$.

CRP is known as an indicator of a first stroke ever $[3,4]$. There is some data indicating a relationship between an elevated CRP and worse outcome [5,6], long-term mortality after ischemic stroke (IS) [7] or further ischemic events in a first-ever transient ischemic attack or stroke patients with intracranial large-artery occlusive disease [8]. CRP level is associated with larger infarct volume [9]. It is debatable whether CRP is a nonspecific marker or rather plays an active role during inflammatory processes. Some studies performed on animal models suggest that CRP is not only a causal factor for stroke. CRP exerts a local effect on endothelial cells and promotes platelet adhesion and subsequent thrombus formation [10]. It affects blood/brain stability [11] and exacerbates the injuries of tissues in ischemic necrosis during a stroke [12]. Adult rats subjected to middle cerebral artery occlusion and then treated with human CRP developed significantly larger cerebral infarcts, unlike those receiving human serum albumin [13].

$\mathrm{S} 100 \mathrm{BB}$ protein is derived from astroglia and can be detected in blood as a result of glial cells destruction caused by ischemia [14]. In addition, S100BB protein can be considered

\footnotetext{
${ }^{1}$ Department of Neurology, Medical University of Lublin, Poland

${ }^{2}$ Department of Medical Chemistry, Medical University of Lublin, Poland

${ }^{3} 1^{\text {st }}$ Department of Radiology, Medical University of Lublin, Poland

${ }^{4}$ Department of Neurosurgery, Medical University of Lublin, Poland

${ }^{5}$ Institute of Psychology, University of Marie Curie-Skłodowska, Poland
} 
as an indicator of blood-brain barrier (BBB) dysfunction during IS [15]. S100BB protein is involved in a variety of intracellular and extracellular functions: regulation of protein phosphorylation, transcription factors, calcium homeostasis, the dynamics of cytoskeleton constituents, cell growth and differentiation $[14,16]$.

\section{AIM}

The aim of our study was to discover whether CRP contributes to releasing S100BB protein into the blood during the acute phase of ischemic stroke (IS). This might shed more light on the mechanisms of CRP activity during IS.

\section{MATERIAL AND METHODS}

Sixty eight patients admitted to the Stroke Unit at the Department of Neurology of the Medical University of Lublin were enrolled into the study in advance. All patients (and family members if necessary) received full written and oral information regarding all study procedures and signed an informed consent. The local ethics committee of the Medical University of Lublin, Poland, in accordance with current legislation on this field, approved the protocol as well as the details of the informed consent. The inclusion criteria were: (a) diagnosis of IS based on history, physical examination and computed tomography (CT)-scan performed upon admission to the hospital, (b) admission to the hospital within the first 24 hours of the onset of neurological focal symptoms.

The exclusion criteria were: (a) regression of neurological symptoms within 24 hours of the onset (Transient Ischemic Attack, TIA) (b) History of central nervous system diseases (epilepsy, dementia, brain tumor) (c) Time of hospitalization shorter than 10 days (d) Hemorrhagic transformation of ischemic focus.

There were fourteen patients that were excluded due to the following factors: 4 of them were TIA patients, 6 had been hospitalized for less than 10 days, and hemorrhagic transformation was detected in 3 patients; one patient died on the fourth day after the stroke onset. The final study group consisted of 54 patients, with the mean age of 72.13 years [SD=11.75], 28 women and 26 men. During the first four days after the stroke onset, anti-hypertensive drugs were not administrated to the patients according to the standard protocol. In case blood pressure was higher than 220/120, Captopril was administered orally. There was no need to administer osmotic agents, such as mannitol, since they could disrupt the blood brain barrier and affect the findings of the study.

The control group consisted of 38 patients with crural varices. The control group was matched with the study group on the basis of their sex and age.

Neurological examination was performed on the following days: the $1^{\text {st }}, 3^{\text {rd }}, 5^{\text {th }}$ and $10^{\text {th }}$ day following the hospital admission, based on the scale of neurological deficits: National Institute of Health Stroke Scale (NIHSS) and functional disability scales: the Barthel Index (BI) and Modified Rankin Scale (mRS). After 3 months, 40 patients were examined by BI and mRS. Twelve patients died within 3 months of hospitalization while two patients remained out of reach.
A CT-scan was performed on days 1 and 10 after hospitalization, without contrast scanning, using a 64-row multidetector CT (LightSpeed VCT with workstation Advantage Window 4.3). Scans were $2.5 \mathrm{~mm}$ thick in posterior fossa and $5 \mathrm{~mm}$ in other areas of the brain. The volume and mean density of ischemic focus were measured by the planimetric method using an additional workstation for measurement infarct volume in 3D.

Venous blood samples were obtained during the first 24 hours after the stroke onset and on days 3, 5 and 10 after the symptoms started to appear. After centrifugation, the serum was stored at $-60^{\circ} \mathrm{C}$ for a maximum of 8 months. Commercially available enzyme-linked immunosorbent assay (ELISA) kits were used to evaluate serum S100BB (CanAg Diagnostics AB, Gothenburg, Sweden) and CRP (R\&D Systems Inc.Minneapolis, USA) according to the manufacturer's instructions. The detection limit of S100BB protein and CRP assay was $10 \mathrm{pg} / \mathrm{mL}$. All values below the detection limit were rendered zero and were not used in calculations. The optical density was determined by using a microplate reader set to $450 \mathrm{~nm}$.

Statistical analysis was performed using the SPSS (Statistical Package for Social Sciences) software for Windows (Version 17.0). For descriptive analyses, we provided median values, mean, standard deviation and percentiles. Statistical differences between the dependent groups were calculated using the Friedman rank test and Wilcoxon signed-ranks test. Comparison between groups with a low and high level of mean CRP was performed using the Mann-Whitney U test. Correlations between CRP, S100BB protein and clinical scales were evaluated by Spearman non-parametric correlation (rs). Linear regression analyses were used to predict the clinical status based on the level of CRP and S100BB protein. The level of significance was set at $p<0.05$. The mean level of CRP and S100BB protein was calculated as the total amount of CRP or S100BB protein measured on 1,3,5 and 10 days after stroke onset divided by the number of days.

\section{RESULTS}

CRP serum levels fluctuated within the acute phase of stroke. The medium CRP level increased gradually till the $5^{\text {th }}$ day, when they started to decrease. There is a positive correlation between a mean level of CRP and NIHSS on day the $10^{\text {th }}$ after the stroke onset. Also, there is a correlation between CRP and mRS on the $10^{\text {th }}$ day and during the 3 months following the stroke onset $(p<0.01)$. It also correlates negatively with BI assessed 10 days and 3 months after stroke $(\mathrm{p}<0.01)$. In addition, we found a correlation between the mean level of CRP and the volume of ischemic focus (rho=0.491 $\mathrm{p}=0.001$ ).

The authors found out that the level of CRP measured on the $1^{\text {st }}, 3^{\text {rd }}, 5^{\text {th }}$ and $10^{\text {th }}$ day following the disease onset, correlates with the results of NIHSS on day 10. The level of CRP on day 3 correlates the strongest ( $r s=0.55 \mathrm{p}<0.001)$.

The level of CRP measured on $1^{\text {st }}, 3^{\text {rd }}, 5^{\text {th }}$ and $10^{\text {th }}$ day following the disease onset correlates positively with $\mathrm{mRS}$ and negatively with BI after 3 months. The level of CRP measured on day 5 correlates the strongest $(\mathrm{rs}=0.53 \mathrm{p}<0.001)$. 
Using regression analysis for measuring the level of CRP on days 3 and 5 (the strongest correlations), the authors tried to assess the possibility of predicting the outcome after ischemic stroke based on the level of CRP. It showed that the level of CRP on the $3^{\text {rd }}$ and $5^{\text {th }}$ day can predict the outcome on both day 10 (assessed by NIHSS) and in the $3^{\text {rd }}$ month (assessed by BI and mRS). The CRP level on the $3^{\text {rd }}$ day appears to be more useful in this case. Increasing the CRP level by about 1 unit rises the NIHSS score on day 10 about 0.09 points, $\mathrm{mRS}$ after 3 months about 0.02 points and decreases BI after 3 months by about 0.39 points.

S100BB protein was present in the serum of all study subjects during 4 consecutive days of examination but it was also detected in the serum of 21 persons $(55.2 \%)$ of the control group. The highest concentration levels of S100BB protein were found in the serum on the $3^{\text {rd }}$ day after the stroke onset. The median serum S100BB level decreased gradually between the $3^{\text {rd }}$ and $10^{\text {th }}$ after the stroke. The mean level of CRP correlates positively with the mean level of S100BB protein $(\mathrm{p}<0.01)$. A statistically significant difference between CRP and S100BB serum levels was observed on all evaluated days. The correlation coefficient is the strongest between the level of CRP measured on day 3 and the level of S100BB measured on day $5(\mathrm{rho}=0.523, \mathrm{p}<0.001)$ (Figure 1) Additionally, we found a statistically significant difference in groups of low and high level of CRP. In the first group with the level of CRP $0.51-24.68 \mathrm{mg} / \mathrm{dL}$ the level of S100BB protein was lower than in the second group

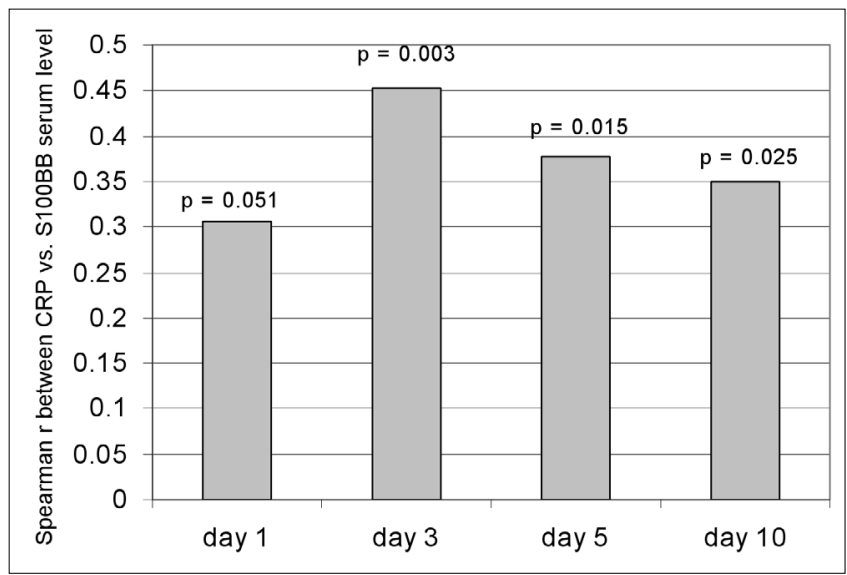

FIGURE 1. The correlation coefficients between CRP and S100BB serum levels on each evaluated days. The strongest correlation $(r=0.453)$ was noticed on day 3 of stroke. Spearman rank correlation.

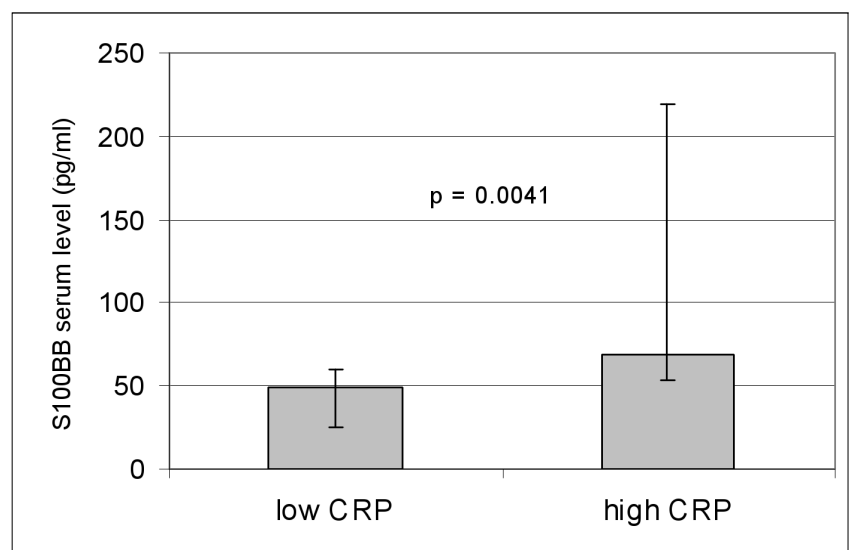

FIGURE 2. Mean S100BB serum level in patients with low and high mean CRP serum level. Mann-Whitney test.
(24.69-209mg/dL). (Figure 2) The mean volume of ischemic focus was smaller in the group with a low level of CRP than in the group with a high level $(\mathrm{Z}=-3.231, \mathrm{p}=0.001)$.

\section{DISCUSSION}

The findings of this study suggest that CRP can show the outcome of the ischemic stroke. Measuring the CRP level on the $3^{\text {rd }}$ day after the stroke gives the most accurate readings that further determines the clinical severity on the $10^{\text {th }}$ day and 3 months after the onset of the disease. The higher the level, the worse clinical conditions, as measured by scales of neurological deficit (NIHSS) and functional status (BI, $\mathrm{mRS}$ ). These results confirm the previously reported findings.

CRP as a general indicator of inflammation reflects the intensity of inflammatory pathways which are engaged during ischemic stroke. However, the results of our study suggest an active role of CRP during processes within ischemic focus. The mean level of CRP correlates not only with the severity of clinical status but also with the size of ischemic focus. We found that CRP can have an influence on the serum $\mathrm{S} 100 \mathrm{BB}$ protein level which is supposed to be a marker of the destruction of BBB. The level of CRP correlates with concentrations of S100BB protein in all evaluated days and the mean concentration of CRP correlates with mean concentrations of S100BB protein. Comparison of the groups with a low and high mean concentration of CRP shows that CRP determines the level of released S100 BB protein. These findings can indirectly indicate that CRP can be engaged in processes which influence the permeability of BBB. It may support earlier observations made on animals. It also shows that the role of CRP during ischemic process is still not clearly defined.

\section{CONCLUSIONS}

Assessing the CRP level can help predict the clinical outcome after ischemic stroke. Its correlations with S100BB protein can suggest that CRP has an active, detrimental role during ischemic processes. The findings of this study go along the ones made on animal models. CRP can be considered as a valuable target for protective treatment during IS.

\section{REFERENCES}

1. Black S, Kusher I, Samols D. C-reactive Protein. J Biol Chem. 2004;279:48487.

2. Thompson D, Pepys MB, Wood SP. The physiological structure of human C-reactive protein and its complex with phosphocholine. Structure. 1999;7:169-77.

3. Elkind MS. Inflammatory mechanisms of stroke. Stroke. 2010;41(Suppl. 10):S3-8.

4. Genest J. C-reactive protein: risk factor, biomarker and/or therapeutic target? Can J cardiol. 2010;26(Suppl. A):41A-44A.

5. Muir KW, Weir CJ, Alwan W, et al. C-reactive protein and outcome after ischemic stroke. Stroke. 1999;30:981-5.

6. Welsh P, Barber M, Langhorne P, et al. Associations of inflammatory and haemostatic biomarkers with poor outcome in acute ischaemic stroke. Cerebrovasc Dis. 2009;27:247-53.

7. Shantikumar S, Grant PJ, Catto A, et al. Elevated C-reactive protein and long-term mortality after ischaemic stroke: relationship with markers of endothelial cell and platelet activation. Stroke. 2009;40:977. 
8. Arenillas JF, Alvarez-Sabin J, Molina CA, et al. C-reactive protein predicts further ischemic events in first-ever transient ischemic attack or stroke patients with intracranial large-artery occlusive disease. Stroke. 2003;34:2463-8.

9. Ormstad H, Aass HCD, Lund-Sorensen N, et al. Serum levels of cytokines and $\mathrm{C}$-reactive protein in acute ischemic stroke patients, and their relationship to stroke lateralization, type, and infarct volume. J Neurol. 2011;258:677-85.

10. Grad E, Pachino RM, Danenberg HD. Endothelial C-reactive protein increases platelel adhesion under flow conditions. Am J Physiol Heart Circ Physiol. 2011;301:H730-6.

11. Kuhlmann CR, Librizzi L, Closhen D, et al. Mechanisms of C-reactive protein-induced blood-brain barrier disruption. Stroke. 2009;40:145866.
12. Pepys MB, Hischfield GM, Tennent GA, et al. Targeting C-reactive protein for treatment of cardiovascular disease. Nature. 2006;440:1217-21.

13. Gill R, Kemp JA, Sabin C, Pepys MB. Human C-reactive protein increases cerebral infarct size after middle cerebral artery occlusion in adult rats. J Cereb Blood Flow Metab. 2004;24:1214-8.

14. Sen J, Belli A. S100B in neuropathologic states: the CRP of the brain? J Neurosci Res. 2007;85:1373-80.

15. Wang M, Etu J, Joshi S. Enhanced disruption of the blood brain barrier by intracarotid mannitol injection during transient cerebral hypoperfusion in rabbits. J Neurosurg Anesthesiol. 2007;19:249-56.

16. Kleindienst A, Ross Bullock M. A critical analysis of the role of the neurotrophic protein S100B in acute brain injury. J Neurotrauma. 2006;23:1185-200.

Corresponding author

Joanna Bielewicz

8 Jaczewskiego Str., 20-954 Lublin

E-mail: j.bielewicz@op.pl

tel: $+4881724-47-20$ 